\title{
Dry eye disease among Glaucoma patients on topical hypotensive medications, in a tertiary hospital, Ethiopia
}

Miraf Sahlu and Abeba T. Giorgis ${ }^{*}$

\begin{abstract}
Background: Dry eye disease is a multifactorial disease; causing various ocular symptoms with potential damage to the ocular surface. Applying hypotensive eye drops are presumed to initiate or exacerbate existing dry eye disease. The purpose of this study was to determine the frequency of signs and symptoms and severity of dry eye disease among glaucoma patients on topical hypotensive medications and controls.

Methods: A cross-sectional comparative study, involving 320 glaucoma patients and controls. Ocular Surface Disease Index (OSDI) symptoms score and Schirmer, tear breakup time and corneal staining tests were used to assess dry eye disease. Data was analyzed using SPSS version 24 software; $p$-value less than 0.05 was considered as statistically significant.

Results: Among the 160 study glaucoma patients, the mean duration of topical hypotensive medication use was $5.2 \pm 5.21$ years (range, 4 months - 32 years). Mild to severe level of OSDI score was found in 122 (76\%) glaucoma patients and in $137(86 \%)$ controls $(p=0.033)$. Mild to sever abnormal clinical tests in the glaucoma patients and control, respectively, were $106(66 \%)$ vs $80(50 \%)$ corneal staining $(p=0.045), 79(49 \%)$ vs $72(45 \%)$ TBUT ( $p=0.021)$, and $91(57 \%)$ vs $83(52 \%)$ Schirmer test $(p=0.242)$. Test results at the level of sever: $2(1 \%)$ vs $0(0 \%)$ corneal staining, 50 (31\%) vs 39 (24\%) TBUT and 65 (41\%) vs 60 (38\%) Schirmer test in the glaucoma patents and controls, respectively. Corneal staining and TBUT had correlation with the number of drugs ( $p=0.004$ and 0.031 , respectively), and more relationship of the two tests with total number of drops applied per day $(p=0.01$ and $p<0.001$, respectively). Patients on pilocarpine and timolol had more corneal staining and lower TBUT [ $(p=0.011$ and $p<0.001)$ and $(p=0.04$ and 0.012$)$, respectively].

Conclusions: The study has identified glaucoma patients to be more affected by dry eye disease than nonglaucoma patients, and presence of significantly lower TBUT and higher corneal staining in the glaucoma patients on multidrops and multidose per day. We recommend consideration of evaluation and management of DED for glaucoma patients on multidrops and multidose hypotensive medications.
\end{abstract}

Keywords: Dry eye disease, Glaucoma, Hypotensive medication

\footnotetext{
* Correspondence: abebatgiorgis@yahoo.com

Department of Ophthalmology, Menelik II Hospital, School of Medicine, College of Health Sciences, Addis Ababa University, P.O.Box 8079, Addis Ababa, Ethiopia
} 


\section{Study design}

Impact of hypotensive drops on dry eye disease: A crosssectional comparative study involving glaucoma patients on hypotensive drops and control.

\section{Background}

Dry eye disease (DED) or ocular surface disease (OSD) is a multifactorial disease of the eye. The disease is characterized by failure to produce high quality or sufficient amounts of tears to moisturize the ocular surface. The disease causes symptoms of discomfort, visual disturbance and tear film instability with potential damage to the ocular surface [1]. The prevalence of DED ranges from 5 to $50 \%$ globally and $6.8 \%$ in USA $[2,3]$. It is more common among older age people and in women than men $(8.8 \%$ vs $4.5 \%)$ [3].

Among the risk factors for DED are ocular and systemic medications [4]. Glaucoma, which mainly affects people age 40 and above, is commonly treated with either single or multiple hypotensive eye drops [5]. It is known that applying hypotensive eye drops over long period of time to either initiate or exacerbate existing dry eye disease. Therefore, glaucoma patients have double risk to be affected by DED [6-10].

Studies have been using structured scoring format including ocular surface disease index (OSDI), standardized patient evaluation of eye dryness (SPEED), and dry eye questionnaire (DEQ-5) to quantify the severity of DED/OSD symptoms [11-13]. Clinical tests that have been used to assess the tear volume, and the extent of ocular surface epitheliopathy are Schirmer test and ocular surface staining using fluorescein, rose Bengal and lissamine green, respectively [14].

The diagnosis of DED in glaucoma patients is often overlooked as the focus of management is on controlling the eye pressure; even if, DED has impact on drug adherence and the quality of life [15]. Therefore, the purpose of this study was to assess the frequency of signs and symptoms and severity of dry eye disease among glaucoma patients on topical hypotensive medications and controls.

\section{Methods}

A hospital-based cross-sectional comparative study was conducted at a glaucoma unit and general out patient clinics of the department of ophthalmology, Menelik II tertiary referral hospital, Ethiopia from October 1, 2019 to November 15, 2019. The source populations for this study were all glaucoma patients who received services at the glaucoma unit and ophthalmic patients attending the general out patient clinics. All glaucoma patients who had follow-up visit and new ophthalmic patients (controls) who came for care during the study period were the study population.
Sample size determination and sampling technique

The sample size was calculated using the formula for comparative studies [16]. Taking the proportion from other similar studies [17], it was assumed that the minimum prevalence of DED among glaucoma patients to be $39 \%$ and that of non-glaucoma patients $25 \%$. The significant level was taken as $95 \%$ with alpha error set to $5 \%$ and power of the study was set at $80 \%$. The ratio of the cases to the control was taken as one. Using these parameters, the required sample size came to be 160 for each group.

\section{Recruitment of study participates}

All glaucoma participants were recruited consecutively using their charts during regular scheduled glaucoma unit appointment days. The controls were new patients attending the general ophthalmic outpatient clinics of the department; similarly, they were recruited consecutively using their charts from a triage room. Ophthalmic/general nurses measured both visual acuity and intraocular pressure, and document on the patient's chart prior getting eye care service form physicians. While the patients were waiting in the waiting area, they were screened for eligibility based on the inclusion and exclusion criteria using their charts, history and examination under slit lump microscope. The selected patients were briefed about the purpose of the study, the questionnaires and the clinical tests, and then requested for willingness to participate. Eye that fulfilled the inclusion criteria was selected, and when both eyes met the inclusion criteria, the right eye was taken.

\section{Inclusion and exclusion criteria}

Inclusion criteria for the glaucoma patients were: 1) age above 40,2) diagnosed with any type of glaucoma and 3) on topical hypotensive medication ( $\mathrm{s}$ ) for three months and above. Exclusion criteria were: 1) use of any nonhypotensive topical drops within the previous 3 months, 2) active or recent ocular infection, 3) presence of immune-compromising disease, 4) lid abnormality such as ectropion, entropion, lagophthalmous, trichiasis and blepharitis, 5) prior lid or ocular surgery and 6) any ocular surface lesions. The controls were also age 40 and above and the exclusion criteria were the same as for the glaucoma patients.

\section{Data collection and analysis}

The participants were given OSDI questionnaire to be filled by themselves or by assistant (accompany person or ophthalmic nurse) if they couldn't do it. The OSDI questionnaire (Allergan Inc., Irvine, CA), which is originally written in English was translated into a national language, Amharic, for the purpose of this study, and was 
verbally translated for those who speak other languages by accompany persons or others.

When answering the questionnaires if the participant didn't do any of the activities in the questionnaire, for example doesn't watch TV, he / she didn't have to answer the specific question related to watching TV. The score was calculated out of the total questions answered by the participants not by all the questions asked. Each question of OSDI is graded from 0 to 4: 0 indicating none of the time, 1 some of the time 2 half of the time, 3 most of the time, and 4 all of the time.

The participants were also interviewed about their sociodemographic background. For the glaucoma patients, information on hypotensive drops including duration of use, type, number and frequency of drops application was obtained using an interviewer administered structured questionnaire. Patients' charts were used to retrieve information on the type of medication(s) if patients were not sure during the interview, and the diagnosis and stage of glaucoma. Then patients of both groups under went through the three standard clinical tests. The tests were performed in the following order.

\section{Schirmer I test (without anesthesia)}

Each participant was asked to look up and the lower eyelid was drawn gently downward on the temporal side. Rounded bent end of a sterile Schirmer strip (Iscon Surgical Ltd., An ISO 9001, Marudhar, India) was hooked in the inferior fornix over the junction of the temporal and central one third of the lower eyelid margin with care taken to avoid touching the cornea; then the patient was asked to close his/her eyes. After $5 \mathrm{~min}$, the strip of filter paper was removed and the amount of wet filter paper was measured and recorded in millimeters. If the tear front moved unevenly, it was measured from the notch to the middle of the diagonal line. Only whole numbers rounded up to the next whole number were recorded if the tear front is at or greater than the half-millimeter mark.

\section{Tear break up time (TBUT)}

One drop of topical anesthesia - tetracaine, was instilled and the patient was asked to close his/ her eyes. After 1 min the patient was instructed to look up to apply a fluorescein sodium ophthalmic strip into the inferior fornix, blink 5 times and then to hold the eyes open. The cornea was scanned with slit lamp microscope using a cobalt blue filter at $10 \mathrm{X}$ magnification. A dry area was indicated by the appearance of a black spot or line. The time in seconds between the last blink and the appearance of black spot was recorded using stopwatch as a tear film break-up-time.

\section{Corneal and conjunctival fluorescein staining}

Under slit-lamp microscope with 10X magnification, extent of staining of the conjunctiva and cornea was assessed and graded against the standard Oxford chart [18].

Operational definitions: OSDI questionnaire was graded as normal $0-12$, mild to moderate $13-32$ and severe 33-100. The value of OSDI was calculated as sum of the severity for all questions answered divided by $4 \mathrm{X}$ total number of questions answered.

Schirmer test: normal $>=10 \mathrm{~mm}$, mild to moderate 6$9 \mathrm{~mm}$ and severe $0-5 \mathrm{~mm}$. The TBUT graded as normal $>=10 \mathrm{~s}$, mild to moderate $5-9 \mathrm{~s}$ and severe $<5 \mathrm{~s}$. Corneal fluorescein staining: normal if 0 (no staining), mild I or II, moderate III and Severe IV or V. Non normal grades were considered as abnormal results.

Data quality control and analysis: At the end of each day, all of the collected data was reviewed and checked for completeness and entered into SPSS (Statistical Package for the Social Science; SPSS Inc., Chicago, IL) version 24 software for data analysis. Descriptive statistics such as frequencies, percentages and mean were used to summarize the demographic data, drug duration, type of medications and OSDI score and results of the three clinical tests. Pearson's correlation, t-test and oneway ANOVA were utilized for further analysis when indicated. $P$-value $<0.05$ was considered to be statistically significant.

\section{Ethical consideration}

The study was carried out in accordance with the tenets of the Declaration of Helsinki, and got ethical approval from the research and publication ethics committee of the Department of Ophthalmology, College of Health Sciences, Addis Ababa University. Written informed consent was obtained from each participant involved in the study. Patients with tests indicating dry eye were informed; the results were documented on their chart to be seen and managed by the treating physicians.

\section{Result}

A total of 320 patients involved in the study, 160 glaucoma and 160 control patients. The details of sociodemographic characteristics of both groups are summarized in Table 1. The mean \pm SD age was $62.2 \pm$ 11.58 (range, 41-87) years in the glaucoma patients and $61.4 \pm 10.76$ (range, 40-90) years in the controls. Male to female ratio was similar in both groups (1.1:1 and 0.9: 1 , respectively).

Primary open angle glaucoma was the commonest diagnosis $(87,54 \%)$ followed by Pseudoexfoliative glaucoma $(65,41 \%)$. The glaucoma patients had been using 7 types of hypotensive eye drops; none of which were preservative free. The types of drops were timolol (53\%), 
Table 1 Sociodemographic Characteristics of the Glaucoma Patients and Controls

\begin{tabular}{|c|c|c|c|}
\hline Characteristic & $\begin{array}{l}\text { Glaucoma Patients } \\
\text { No (\%) }\end{array}$ & $\begin{array}{l}\text { Controls } \\
\text { No }(\%)\end{array}$ & $P$ Value* \\
\hline \multicolumn{4}{|l|}{ Age (years) } \\
\hline $40-50$ & $32(20)$ & $28(18)$ & \\
\hline $51-60$ & $40(25)$ & $48(30)$ & \\
\hline $61-70$ & $53(33)$ & $59(37)$ & 0.51 \\
\hline $71-80$ & $27(17)$ & $19(12)$ & \\
\hline$>80$ & $8(5)$ & $6(4)$ & \\
\hline \multicolumn{4}{|l|}{ Sex } \\
\hline Male & $85(53)$ & $76(48)$ & 0.31 \\
\hline Female & $75(47)$ & $84(53)$ & \\
\hline \multicolumn{4}{|l|}{ Address } \\
\hline Urban & $112(74)$ & $136(85)$ & 0.18 \\
\hline Rural & $48(21)$ & $24(15)$ & \\
\hline \multicolumn{4}{|l|}{ Occupation } \\
\hline Indoor & $112(70)$ & $127(80)$ & 0.055 \\
\hline Outdoor & $48(30)$ & $33(21)$ & \\
\hline \multicolumn{4}{|l|}{ Educational status } \\
\hline Illiterate & $45(28)$ & $23(14)$ & \\
\hline Primary & $55(34)$ & $29(18)$ & $<0.001$ \\
\hline Secondary & $24(15)$ & $47(29)$ & \\
\hline College \& above & $36(23)$ & $61(38)$ & \\
\hline
\end{tabular}

*Paired sample t-test was used to compare the categorical variables

timolol and dorzolamide fixed-combination (41\%), pilocarpine (36\%), latanaprost (12\%), brimonidine (25\%), betaxolol (2\%) and dorzolamide (1\%). The mean \pm SD duration of topical medication use was $5.2 \pm 5.21$ years (range, 4 months - 32 years). The minimum and maximum frequency of drop(s) per day was 1 and 6. Two and 5 drops per day were the common frequent regimes (51 and 34\%, respectively).

The mean \pm SD of DED symptom with OSDI score was $30.8 \mathrm{SD} \pm 22.9$ (range, 0-100) in the glaucoma patients, and $36.1 \pm 21.3$ (range, $0-86$ ) in the control, $p=$ 0.033 . Mild to severe level of OSDI value was detected in $122(76 \%)$ glaucoma patients and 137 (86\%) controls. The level was severe in $69(43 \%)$ glaucoma patients and 85 (53\%) controls, $p=0.028$.

Table 2 shows the results of the 3 clinical tests among the glaucoma and control patients; a paired sample $t$ test comparing the mean values of the two groups for each test. The difference between the glaucoma and control participants with regard to corneal staining and TBUT was statistically significant. Abnormal (mild sever) corneal staining was detected in $106(66 \%)$ glaucoma patients and $80(50 \%)$ controls, $p=0.045$. Tear breakup time was abnormal in 79 (49\%) glaucoma patients and $72(45 \%)$ controls, $p=0.021$. While Schirmer
Table 2 Clinical Tests of Dry Eye Disease of Glaucoma Patients and Controls

\begin{tabular}{|c|c|c|c|c|c|}
\hline \multirow[t]{2}{*}{ Test } & \multicolumn{2}{|c|}{ Glaucoma Patients } & \multicolumn{2}{|c|}{ Controls } & \multirow{2}{*}{$\begin{array}{l}P \\
\text { Value* }\end{array}$} \\
\hline & No & $\%$ & No & $\%$ & \\
\hline \multicolumn{6}{|l|}{ Corneal Staining } \\
\hline Normal & 54 & 33 & 80 & 50 & 0.045 \\
\hline Mild & 90 & 56 & 66 & 41 & \\
\hline Moderate & 14 & 9 & 14 & 9 & \\
\hline Severe & 2 & 1 & 0 & & \\
\hline \multicolumn{6}{|l|}{ TBUT } \\
\hline Normal & 81 & 51 & 88 & 55 & 0.021 \\
\hline Mild to moderate & 29 & 18 & 33 & 21 & \\
\hline Severe & 50 & 31 & 39 & 24 & \\
\hline \multicolumn{6}{|l|}{ Schirmer } \\
\hline Normal & 69 & 43 & 77 & 48 & 0.242 \\
\hline Mild to moderate & 26 & 16 & 23 & 14 & \\
\hline Severe & 65 & 41 & 60 & 38 & \\
\hline \multicolumn{6}{|c|}{ Total number of abnormal tests } \\
\hline Zero/all normal & 21 & 13 & 39 & 25 & 0.034 \\
\hline One & 41 & 26 & 44 & 28 & \\
\hline Two & 59 & 37 & 40 & 25 & \\
\hline Three & 39 & 24 & 37 & 23 & \\
\hline
\end{tabular}

test was abnormal in 91 (57\%) glaucoma patients and 83 (52\%) controls, $p=0.242$. The glaucoma patients had higher number with 2 or 3 abnormal clinical test results (98 vs 77), while the controls had more normal results in all the 3 clinical tests than the glaucoma patients (39 vs 21).

Pearson's correlation coefficient was used to see any correlation between the OSDI results and the 3 clinical tests. For the glaucoma patients, the OSDI results had statistically significant correlation with the overall number of abnormal tests $(p=0.036)$ (Fig. 1), and a strong correlation with Schirmer test $(p=0.009)$. On the other hand, the correlation of OSDI score with corneal staining and TBUT was not significant $(p=0.149$ and 0.126 respectively).

The results of OSDI questionnaire and the clinical tests were dichotomized into normal versus abnormal as seen in Table 3 for the glaucoma Patients. The analysis showed that those who had abnormal test results were 3 times more likely to report DED symptoms than those with normal results in all the three clinical tests (odd ratio $=3.00$ with $95 \%$ CI $1.5-7.9, p=0.022$ ). For the control, the OSDI results didn't correlate significantly with the number of abnormal tests $(p=0.201)$.

One-way ANOVA analysis was utilized to find out any difference among the different sociodemographic characteristics and OSDI questionnaires score and the 


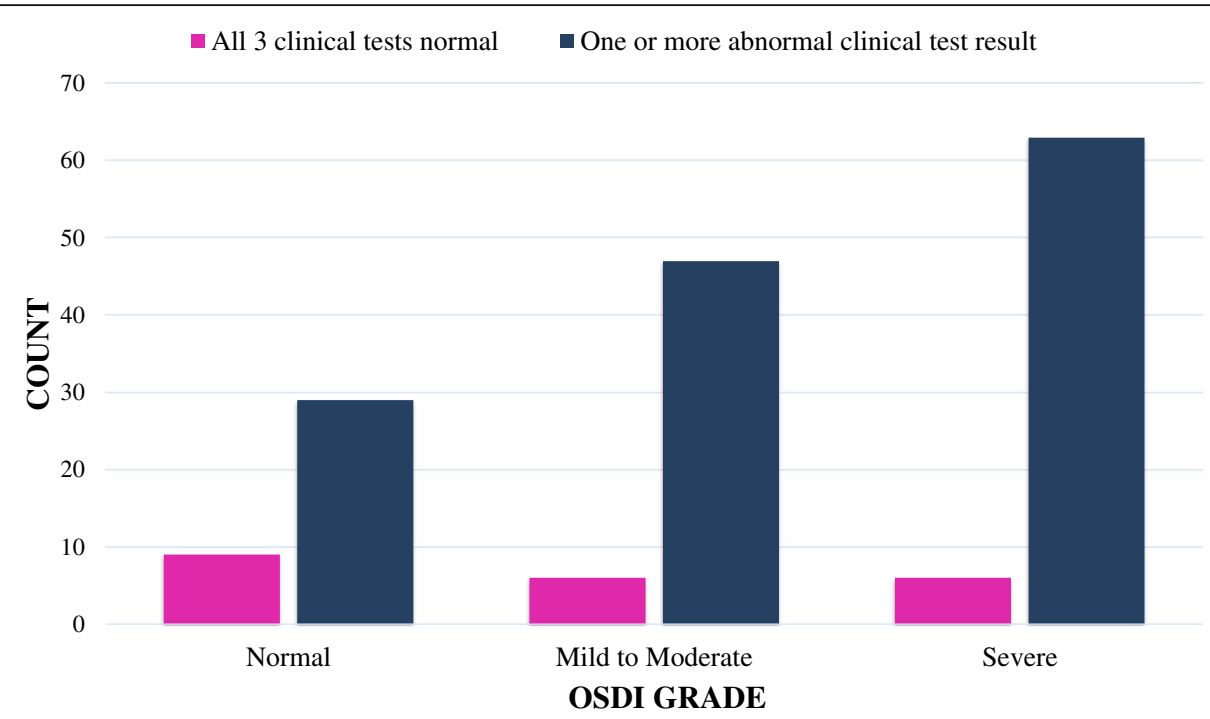

Fig. 1 Relationship between OSD symptoms \& clinical test results in glaucoma patients

clinical test results in both the glaucoma patients and controls, which showed no statistically significant difference. There was no significant difference among the different educational levels with the result of the clinical tests and the OSDI score; except the TBUT result of the glaucoma patients which had mean value of $7.6 \pm 4.6 \mathrm{~s}$ vs $11 \pm 6.0 \mathrm{~s}(p=0.03)$ in those who can't read and write versus those with college and above educational level, respectively.

To see the effect of polypharmacy on abnormal test results, the glaucoma patients were divided and analyzed as those applying only one type of hypotensive medication and those applying 2 or more medications. The analysis showed a statistically significant mean difference in total number of abnormal tests between the two groups, $1.5 \pm 0.96$ vs $1.9 \pm 0.96, p=0.013$.

The mean $\pm S D$ number of drops applied per day in early, moderate and advanced stage of glaucoma were $2.5 \pm 1.14,3.0 \pm 1.37,3.7 \pm 1.46$, respectively. One-way ANOVA analysis revealed the presence of higher mean corneal staining in the advanced stage as compared to the early and moderate stage of glaucoma, $1.4 \pm 1.13$ vs $0.6 \pm 0.66(p<0.001)$ and $1.4 \pm 1.13$ vs $1.0 \pm 0.90, p=$ $0.04 \overline{7}$, respectively. Additionally, TBUT was lower $(7.6 \pm 5.1$ vs $10.7 \pm 4.97 \mathrm{~s}, p=0.009)$ and the number of abnormal tests was higher $(1.9 \pm 0.94$ vs $1.4 \pm 0.97, p=$ $0.014)$ in advanced stage than early and moderate stage of glaucoma. But the stage of glaucoma didn't correlate significantly with OSDI score and the Schirmer test. There was no significant difference between the types of glaucoma (POAG, PXG, OHT) and the OSDI score and the number of abnormal tests.

Pearson's correlation was also used to see if there was a relationship of the number of hypotensive drugs, total number of drops applied per day and drug duration in years with the 3 clinical tests and the OSDI score. The corneal staining and TBUT had significant correlation with the number of drugs $(p=0.004$ and 0.031 , respectively); even more significant relationship of the two tests with total number of drops applied per day ( $p=$ 0.01 and $p<0.001$, respectively). Duration of drop application had no significant association with corneal staining and TBUT. Figure 2 shows the relationship between the grades of TBUT and corneal staining with the number of drops applied per day. Schirmer test and OSDI score had no statistically significant correlation with the number of drugs, drops per day or duration of medication use.

T-test was used to compare those applying one type of drug to those on other drops in relation to the OSDI

Table 3 Agreement between OSD symptoms and clinical test results in glaucoma patients

\begin{tabular}{|c|c|c|c|c|c|c|c|}
\hline & & \multicolumn{2}{|l|}{ TBUT } & \multicolumn{2}{|c|}{ SCHIRMER } & \multicolumn{2}{|c|}{ CORNEAL STAINING } \\
\hline & & Normal & Abnormal & Normal & Abnormal & Normal & Abnormal \\
\hline \multirow[t]{2}{*}{$\overline{\text { OSDI }}$} & Normal & 25 & 14 & 21 & 18 & 12 & 27 \\
\hline & Abnormal & 56 & 65 & 47 & 74 & 43 & 78 \\
\hline \multicolumn{2}{|c|}{$\begin{array}{l}\text { OR: } 95 \% \mathrm{Cl} \\
P \text { value }\end{array}$} & \multicolumn{2}{|c|}{$\begin{array}{l}2.0(0.98-4.3) \\
0.039\end{array}$} & \multicolumn{2}{|c|}{$\begin{array}{l}1.84(0.88-3.8) \\
0.10\end{array}$} & \multicolumn{2}{|c|}{$\begin{array}{l}0.8(0.37-1.7) \\
0.04\end{array}$} \\
\hline
\end{tabular}



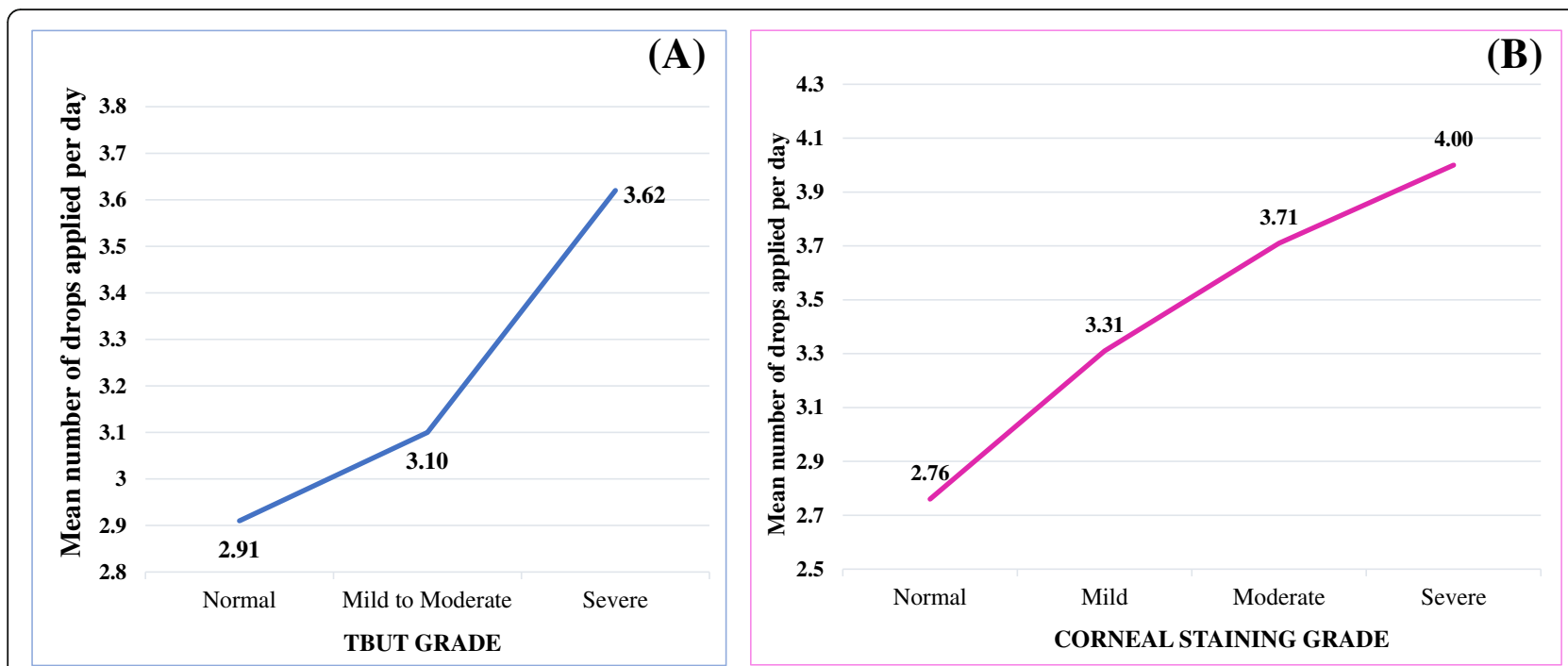

Fig. 2 Relationship between mean number of drops applied per day with Tear Break Up Time (a) \& corneal staining (b)

and the 3 clinical tests. Those on pilocarpine had statistically significant lower TBUT and more corneal staining results than those not applying the drug ( $p=0.011$ and $p<0.001$, respectively). Besides, those on timolol or combination of timolol and dorzolamide had statistically significant abnormal test results than those not applying the drug ( $p=0.04$ and $p=0.012$ respectively).

\section{Discussion}

This study assessed dry eye disease in glaucoma patients on topical hypotensive drops and controls using OSDI symptoms score and three clinical tests (corneal staining, tear breakup time and Schirmer test).

The similarity in mean age, $62.2 \pm 11.58$ and $61.4 \pm$ 10.76 years, and female sex proportion, 75 (47\%) and 84 (53\%), in the glaucoma patients and controls, respectively, could help to balance the age and sex related ocular surface changes which are the leading risk factors for dry eye disease [1-3].

The mean \pm SD of DED symptom with OSDI score was higher in the controls than the glaucoma patients ( $36.1 \pm 21.3$ vs $30.8 \pm 22.91)$. In addition, both mild to severe and severe level of OSDI were higher in the controls $(137,86 \%$ and $85,53 \%)$ than in the glaucoma patients $(69,43 \%$ and $69,43 \%)$. The controls were new patients who had not received any ophthalmic attention at the hospital; thus, their response to the OSDI questionnaire might be exaggerated to get attention than the glaucoma patients who had been already diagnosed, on treatment and follow-up. On the other hand, the glaucoma patients might be more concerned about their glaucoma than other symptoms. Besides, the OSDI score of the controls has poor correlation with the clinical test results. Such poor correlation was also found in other studies and the potential explanation given were reporting bias by patients, heterogeneity of the disease and at times paucity of symptoms in a subset of patients with severe disease $[19,20]$. On the other hand Barisic et al. has reported mild to sever OSDI in $75 \%$ of 110 glaucoma patients on topical hypotensive medications and in $30 \%$ of 50 controls [21].

Comparing to other study reports on glaucoma patients, our participants in both groups had higher percentage of abnormal OSDI results $(76 \%$ in the glaucoma and $86 \%$ in the controls), and reported more severe symptoms, $43 \%$ glaucoma patients and $53 \%$ controls $[8,9]$.

As depicted in Table 2, the glaucoma patients had more abnormal results in the three clinical tests than the controls (corneal staining $66 \%$ vs $46 \%, p=0.045$, TBUT $49 \%$ vs $45 \%, p=0.021$ and Schirmer $57 \%$ vs $52 \%, p=$ 0.242). These results are in line with a similar crosssectional comparative study by Ramli et al. that reported higher percentage of abnormal tests in the glaucoma group than the control (corneal staining 63\% vs $36 \%$, $p=0.004$ and Schirmer $39 \%$ vs $25 \%, p=0.049$ ) [17].

The glaucoma patients were on 7 different hypotensive eye drops with benzalkonium chloride (BAC) preservative, which are additional risk factors for DED $[6,7,22]$. The higher ocular surface disease signs in the glaucoma patients than the controls can be explained by the toxic effect of BAC preservative and active molecular effect of the medication(s) on the corneal epithelial cells [23]. Moreover, Cha and his colleagues have proven BAC to induce corneal epithelial lysis and even more dysfunction with higher concentration on their experimental study on cultured rabbit corneal epithelial cells [24]. 
Considering the severity of the clinical tests in this study, the proportion of severe results in the glaucoma patients and the controls were Schirmer test $41 \%$ vs $38 \%$, TBUT $31 \%$ vs $24 \%$ and corneal staining $1 \%$ vs $0.0 \%$. There is difference and similarity when these severity results are compared with a cross sectional study on the prevalence of ocular surface disease in 101 glaucoma patients by Leung et al. that reported the presence of severe tear deficiency: Schirmer test in 35\%, TBUT in $65 \%$ and corneal staining in $0 \%$ [8]. The figure variations could be due to the involvement of both eyes and taking the result in at least one eye, while in our study the involvement was only one eye of each patient; still the data are indicative of the extent of DED in glaucoma patients. Trachoma, vitamin A deficiency and higher environmental temperature are the additional risk factors for DED that has to be considered among Africans [25]. This can be considered as one explanation for the higher test results in our study participants.

In this study, even though, OSDI symptom score was higher in the controls, its correlation with the overall number of abnormal clinical tests was significant $(p=$ 0.036) in the glaucoma patients. Besides, those who had abnormal test results were 3 times likely to report symptoms (odd ratio $=3.00$ with $95 \%$ CI $1.5-7.9, p=0.022$ ), which was not the case for the controls (Table 3). This indicates the relevance of testing to confirm the presence of DED in symptomatic patients, and also asking glaucoma patients on topical drops if they have symptoms in order to recognize and treat the disease that has impact on patient quality of life and medication adherence [7]. Considering the age and eye medications related risks for DED, it is reasonable to consider DED evaluation as part of glaucoma patient management.

Depending on the stage of glaucoma, patients with advanced stage were found to apply more number of medications $(3.7 \pm 1.46 \mathrm{SD})$ than those with early and moderate stage of glaucoma. In relation to this, the analysis showed the presence of higher corneal staining, lower TBUT and higher abnormal tests in the advanced stage. Moreover, both corneal staining and TBUT were found to have correlation with increased number of drugs ( $p=0.004$ and 0.031 , respectively) and even more significant correlation with total number of drops applied per day ( $p=0.01$ and $p<0.001$, respectively) (Fig. 2 ). These finding can be explained by the likelihood of increased adverse effects of the medications and/ or their preservative (BAC) on the ocular surface and the quality of tear film, which could be dose and drug number dependent $[15,26]$.

The analysis finding of the presence of association of lower TBUT and more corneal staining with pilocarpine use and drops containing timolo in this study can be supported by an experimental study on the influence of pilocarpine and timolol on human meibomian gland epithelial cells by Zhang et al. that detected the presence of direct effect of these drugs on the morphology, survival and proliferation capacity of the gland cells [27]. Moreover, pilocarpine's tear film $\mathrm{pH}$ lowering effect has been reported by Azuamah et al. [28]. Therefore, based on our findings and those study reports, we may consider direct effect of these drugs on the ocular surface in addition to their preservative.

Limitations of the study: All the participants were using drops with BAC as a preservative and the effect of preservative-free drugs was not seen in this study.

\section{Conclusions}

The study has identified glaucoma patients to be more affected by dry eye disease than non-glaucoma patients, and the presence of significantly lower TBUT and higher corneal staining in the glaucoma patients on multidrops and multidose per day. We recommend consideration of evaluation and management of DED for glaucoma patients on multidrops and multidose hypotensive medications.

\section{Abbreviations}

DED: Dry eye disease; OSDI: Ocular Surface Disease Index; TBUT: Tear break Up Time; SPSS: Statistical Package for the Social Science; PXG: Primary open angle glaucoma (POAG), Pseudoexfoliative glaucoma; OHT: Ocular hypertension

\section{Acknowledgements \\ The authors would like to acknowledge all the participants of the study. The Department of Ophthalmology, School of Medicine, College of Health Sciences, Addis Ababa University is thanked for facilitating to conduct the study and for research granting. All the nurses working at the glaucoma unit and the general out patient clinics of the department at Menelik Tertiary Referral Hospital are acknowledged for being helpful during the date collection.}

\section{Authors' contributions}

MS prepared the study proposal and involved in data collection, analysis and result writing. ATG involved in conception and supervision of the study and preparing the manuscript. Both authors read and approved the final manuscript.

\section{Authors' information \\ MS is senior ophthalmic resident of ophthalmology and ATG is an associate professor and senior glaucoma consultant. Both authors are at the Department of Ophthalmology, School of Medicine, College of health Sciences, Addis Ababa University. \\ Funding \\ The study was supported by research grant for graduate students form the College of Health Sciences, Addis Ababa University. The college had no any role in the design of the study and collection, analysis and interpretation of the data and in writing the manuscript, or decision to publish this manuscript.}

\section{Availability of data and materials}

The datasets used and analyzed during the current study are available from the first author on reasonable request. 


\section{Declarations}

\section{Ethics approval and consent to participate}

The study was undertaken after obtaining ethical clearance from the research and publication ethics committee of the Department of Ophthalmology, School of Medicine, College of Health Sciences, Addis Ababa University. Written informed consent was also obtained from the glaucoma patients and control. The study adhered to the declaration of Helsinki.

\section{Consent for publication}

Not applicable.

\section{Competing interests}

The authors declare that they have no competing interests.

Received: 1 December 2020 Accepted: 18 March 2021

Published online: 30 March 2021

\section{References}

1. Craig JP, Nichols KK, Akpek EK, Caffery B, Dua HS, Joo CK, et al. TFOS DEWS II dry eye definition and classification report. Ocul Surf. 2017;15(3):276-83. https://doi.org/10.1016/j.jtos.2017.05.008

2. Stapleton F, Alves M, Bunya WY, Jalbert I, Lekhanont K, Malet F, et al. TFOS DEWS II dry eye epidemiology report. Ocul Surf. 2017;15(3):334-65. https:// doi.org/10.1016/i.jtos.2017.05.003.

3. Farrand KF, Fridman M, Stillman IÖ, Schaumberg DA. Prevalence of diagnosed dry eye disease in the United States among adults aged 18 years and older. Am J Ophthalmol. 2017;182:90-8. https://doi.org/10.1016/j.ajo.201 7.06.033.

4. Rouen PA, White ML. Dry eye disease prevalence, assessment, and management. Home Healthcare Now. 2018;36(2):74-83. https://doi.org/10.1 097/NHH.0000000000000652

5. Allingham RR, Damji KF, Freedman S, Moroi SE, Rhee D. Principles of Medical Therapy and Management. In: Shields textbook of Glaucoma. 6th ed. Philadelphia: Lippincott Williams \& Wilkins; 2011. p. 389-99.

6. Wong ABC, Wang MTM, Liu K, Prime ZJ, Danesh-Meyer HV, Craig JP. Exploring topical anti-glaucoma medication effects on the ocular surface in the context of the current understanding of dry eye. Ocul Surf. 2018;16(3): 289-93. https://doi.org/10.1016/j.jtos.2018.03.002

7. Zhang X, Vadoothker S, Munir MW, Saeedi O. Ocular surface disease and Glaucoma medications: a clinical approach. Eye Contact Lens. 2019;45(1):118. https://doi.org/10.1097/ICL.0000000000000544.

8. Leung EW, Medeiros FA, Weinreb RN. Prevalence of ocular surface disease in glaucoma patients. J Glaucoma. 2008;17(5):350-5. https://doi.org/10.1097/ JUG.0b013e31815c5f4f.

9. Fechtner RD, Godfrey DG, Budenz D, Stewart JA, Stewart WC, Jasek MC. Prevalence of ocular surface complaints in patients with glaucoma using topical intraocular pressure-lowering medications. Cornea. 2010;29(6):61821. https://doi.org/10.1097//CO.0b013e3181c325b2.

10. Ammar DA, Noecker RJ, Kahook MY. Effects of Benzalkonium chloridepreserved, Polyquad- preserved, and sofZia- preserved topical Glaucoma medications on human ocular epithelial cells. Adv Ther. 2010;27(11):837-45. https://doi.org/10.1007/s12325-010-0070-1.

11. Schiffman RM, Christianson MD, Jacobsen G, Hirsch JD, Reis BL. Reliability and validity of ocular surface disease index. Arch Ophthalmol. 2000;118(5): 615-2. https://doi.org/10.1001/archopht.118.5.615.

12. Ngo W, Situ P, Keir N, Korb D, Blackie C, Simpson T. Psychometric propreties and validation of the standard patient evaluation of eye dryness questionnaire. Cornea. 2013;32(9):1204-10. https://doi.org/10.1097/ICO. Ob013e318294b0co

13. Chalmers RL, Begley CG, Caffery B. Validation of the 5-item dry eye questionnaire (DEQ-5): discrimination across self-assessed severity and aqueous tear deficient dry eye diagnosis. Cont Lens and Anterior Eye. 2010; 33(2):55-60. https://doi.org/10.1016/j.clae.2009.12.010.

14. Foster C.S. Dry Eye Disease (Keratoconjunctivitis Sicca) Workup. https:// emedicine.medscape.com/article/1210417-workup.

15. Kaštelan S, Tomić M, Soldo MK, Salopek-Rabatić J. How ocular surface disease impacts the Glaucoma treatment outcome. Hindawi Publishing Corp BioMed Res Int http://dx.doi.org/https://doi.org/10.1155/2013/696328. 2013
16. Fleiss $L A$, Levin $B$, Cho Paik CM. Determining Sample Sizes Needed to Detect a Difference between Two Proportions. In: Statistical methods for rates and proportions. 3rd ed. New Jersey: John Wiley \& Sons, Inc.; 2003. p. 64-72.

17. Ramli N, Supramaniam G, Samsudin A, Juana A, Zahari M, Choo MM. Ocular surface disease in Glaucoma: effect of polypharmacy and preservatives. Optom Vis Sci. 2015;92(9):e222-6. https://doi.org/10.1097/OPX. 0000000000000542

18. Bron A, Evans VE, Smith JA. Grading of corneal and conjunctival staining in the context of other dry eye tests. Cornea. 2003;22(7):640-50. https://doi. org/10.1097/00003226-200310000-00008.

19. Skalicky SE, Goldberg I, Mccluskey P. Ocular surface disease and quality of life in patients with Glaucoma. Am J Ophthalmol. 2012;153(1):1-9. https:/ doi.org/10.1016/j.ajo.2011.05.033.

20. Schein OD, Tielsch JM, Muñoz B, Bandeen-Roche K, West S. Relation between signs and symptoms of dry eye in the elderly: a population-based perspective. Ophthalmology. 1997;104(9):1395-401. https://doi.org/10.1016/ S0161-6420(97)30125-0.

21. Barisic F, Krolo I, Suic S, Sesar I, Prskalo M, Mandic Z. Prevalence of ocular surface disease in patients with Glaucoma using topical Antiglaucoma medications. J Clin Exp Ophthalmol. 2014;5(02):2. https://doi.org/10.4172/21 55-9570.1000334.

22. Datta S, Baudouin C, Brignole-Baudouin F, Denoyer A, Cortopassi GA. The eye drop preservative Benzalkonium chloride potently induces mitochondrial dysfunction and preferentially affects LHON mutant cells. Invest Ophthalmol Vis Sci. 2017 Apr;58(4):2406-12. https://doi.org/10.1167/ iovs.16-20903.

23. Stewart WC, Stewart JA, Nelson LA. Ocular surface disease in patients with ocular hypertension and glaucoma. Curr Eye Res. 2011;36(5):391-8. https:// doi.org/10.3109/02713683.2011.562340.

24. Cha SH, Lee JS, Oum BS, Kim CD. Corneal epithelial cellular dysfunction from benzalkonium chloride (BAC) in vitro. Clin Exp Ophthalmol. 2004;32(2): 180-4. https://doi.org/10.1111/j.1442-9071.2004.00782.x.

25. Osae AE, Gehlsen U, Horstmann J, Siebelmann S, Stern ME, Kumah DB, Steven P. Epidemiology of dry eye disease in Africa: the sparse information, gaps and opportunities. December, 2016. Elsevier Inc.

26. Chen H, Lin C, Tsai Y, Kao C. Association between Glaucoma medication usage and dry eye in Taiwan. Optom Vis Sci. 2015;92(9):e227-32. https://doi. org/10.1097/OPX.0000000000000667.

27. Zhang Y, Kam WR, Liu Y, Chen X, Sullivan DA. Influence of pilocarpine and timolol on human meibomian gland epithelial cells. Cornea. 2017;36(6):71924. https://doi.org/10.1097/ICO.0000000000001181.

28. Azuamah YC, Esenwah EC, Ikoro NC, Megwas AU, Ugwuoke AU, Ogbonna GI. Effect of topical 1\% Pilocarpine on the ocular tear film pH. Int J Res. 2019;06(07):120-8

\section{Publisher's Note}

Springer Nature remains neutral with regard to jurisdictional claims in published maps and institutional affiliations.

Ready to submit your research? Choose BMC and benefit from:

- fast, convenient online submission

- thorough peer review by experienced researchers in your field

- rapid publication on acceptance

- support for research data, including large and complex data types

- gold Open Access which fosters wider collaboration and increased citations

- maximum visibility for your research: over $100 \mathrm{M}$ website views per year

At BMC, research is always in progress.

Learn more biomedcentral.com/submissions 